\title{
3-Dimensional Chromatography
}

National Cancer Institute

\section{Source}

National Cancer Institute. 3-Dimensional Chromatography. NCI Thesaurus. Code C161769.

The use of three chromatography-based techniques in sequence to separate the various substances in a mixture. 